This is the final peer-reviewed accepted manuscript of:

A. Giorgetti et al., "A Robust Wireless Sensor Network for Landslide Risk Analysis: System Design, Deployment, and Field Testing" in IEEE Sensors Journal, vol. 16, no. 16, pp. 6374-6386, Aug.15, 2016

The final published version is available online at:

https://doi.org/10.1109/JSEN.2016.2579263

Rights / License:

The terms and conditions for the reuse of this version of the manuscript are specified in the publishing policy. For all terms of use and more information see the publisher's website.

This item was downloaded from IRIS Università di Bologna (https://cris.unibo.it/)

When citing, please refer to the published version. 


\title{
A Robust Wireless Sensor Network for Landslide Risk Analysis: System Design, Deployment, and Field Testing
}

\author{
Andrea Giorgetti, Senior Member, IEEE, Matteo Lucchi, Emanuele Tavelli, Marco Barla, Giovanni Gigli, \\ Nicola Casagli, Marco Chiani, Fellow, IEEE, and Davide Dardari, Senior Member, IEEE
}

\begin{abstract}
In this paper we propose a wireless sensor network (WSN) designed for monitoring and risk management of landslides, where data collected by sensors are delivered through the network to a remote unit (RU) for on-line analysis and alerting. To ensure fast deployment, robustness in harsh environments, and very long lifetime, the sensor nodes and the communication protocol have been specifically conceived so that the network is self-organizing, fault tolerant, and adaptive. The WSN has been installed on a landslide located in Torgiovannetto (Italy) for an experimental campaign of several months where performance metrics, such as radio link and path statistics as well as battery levels, have been collected. These metrics demonstrated the effectiveness of the network protocols to manage self-organization, node failures, low link quality and unexpected battery depletion. With negligible human intervention during the pilot experiment, the WSN revealed a very high level of robustness, which makes it suitable to monitor landslides in critical scenarios.
\end{abstract}

Index Terms-Energy efficiency, deployment, field testing, internet-of-things, landslide monitoring, wireless sensor network.

\section{INTRODUCTION}

$\mathbf{I}$ NTERNET-OF-THINGS (IoT) is the emerging idea of a globally interconnected continuum of devices and things envisaging a plethora of heterogeneous objects interacting with the physical environment. A promising application of IoT is environmental monitoring for public protection and disaster relief, especially challenging because of harsh operating conditions and difficulty and cost of human intervention for deployment and maintenance [1]-[15].

A. Giorgetti, M. Chiani, and D. Dardari are with the Department of Electrical, Electronic, and Information Engineering "Guglielmo Marconi" (DEI), University of Bologna, via Venezia 52, 47521 Cesena (FC), Italy, (email: $\{$ andrea.giorgetti, marco.chiani, davide.dardari\}@unibo.it).

M. Lucchi and E. Tavelli are with Winet Srl, 47521 Cesena (FC), Italy, (e-mail: \{mlucchi, etavelli\}@winetsrl.com).

M. Barla is with the Department of Structural and Geotechnical Engineering, Politecnico di Torino, Corso Duca degli Abruzzi 24, 10129 Torino, Italy, (e-mail: marco.barla@polito.it).

G. Gigli and N. Casagli are with the Earth Science Department, Università di Firenze, via La Pira 4, 50121 Firenze, Italy, (e-mail: \{giovanni.gigli, nicola.casagli\}@unifi.it).

This work was presented in part at the IEEE Int. Conf. on Wireless and Mobile Computing, Networks and Comm. (WiMob 2014), workshop on Emergency Networks for Public Protection and Disaster Relief (EN4PPDR 2014), Larnaca, Cyprus, Oct. 2014. This research was supported in part by the Ministero dell'Istruzione, dell'Università e della Ricerca (MIUR) under the PRIN 2009 project "Integration of monitoring and numerical modeling techniques for early warning of large rockslides" (grant no. 2009A85F98), and in part by the European project EuroCPS (grant no. 644090) under the H2020 framework.
Sustainable land management and development is an essential key to reduce the negative impacts of natural disasters. For this reason, landslide analysis and mapping have received an ever increasing attention in order to identify relevant factors and physical phenomena causing ground movements, such as rockfalls, deep failure of slopes and shallow debris flows, and to finally predict the landslide hazard based on such a relationship. Many factors are necessarily considered for landslide risk analysis, thus it is important to collect, store, manipulate, display, and analyze a large amounts of spatially referenced data which can be handled fast and effectively.

Remote sensing techniques are so far the most employed for landslide hazard assessment and analysis [16]-[22]. Through remote sensing, either based on satellite observations or ground based radars, it is possible to reveal how the landscape changed during an event, detect what may have triggered the landslide, and show the process of regeneration and recovery. While remote sensing provides a relatively high degree of spatial resolution, it is expensive and it requires very accurate installation and lengthy calibration procedures. Therefore, to supervise rare phenomena for a very long time, such as rock landslides, WSNs are the natural choice as the cutting edge technology that can quickly respond to rapid changes of relevant physical parameters and send them to a remote center for further elaboration and alerting.

WSNs are typically based on low-cost, low-energy consumption nodes, whose battery is normally not replaced during network lifetime [23]-[27]. Nodes sense the environment and are equipped with radio transceivers which allow them to act as both transmitters and route-and-forward devices. The characteristics of WSNs and their applications make energy conservation and self-organization primary goals. Accordingly, self-organizing and energy-efficient protocols have been developed for data transmission in WSNs [28]-[31].

WSNs for environmental monitoring has attracted an intense research during the last decade [32]-[35]. A network composed of accelerometers to detect and measure vibrations caused by landslides is presented in [36]. In [37], the authors proposed a prototype of an early warning system investigating the whole chain from data gathering up to data analysis and visualization. An integrated system for landslide detection based on a network of geophysical sensors, its design and deployment, is presented in [38]. A critical review of landslides monitoring techniques and a solution based on a WSN can be found in [39]. In [40], the risk associated to slip 
surface movements and their velocities is analyzed through a WSN. The WSN proposed in [41] is used for soil monitoring with real-time measurements at different temporal and spatial granularities. In [42], a distributed system with a large number of sensor nodes, aimed at predicting landslides in the hilly regions of western India, is presented. The adoption of a WSN to monitor a scenario after a landslide disaster is proposed in [43]. The use of different sensor types in deformation monitoring is described in [44]. In [45], the authors developed a WSN for an effective drought forecast and alert.

In developing a WSN for landslides monitoring there are several crucial aspects that need to be considered. In the following, we summarize the most important challenges inspiring our design:

- Long network lifetime is required to reduce human intervention and risks, e.g., for battery replacement in hostile environments.

- Landslide detection is a rare event which requires continuous monitoring for very long periods. This makes energy consumption challenging.

- The WSN operates in harsh environments, where node failures may occur unexpectedly. Synchronization and routing algorithms need to be fault tolerant to guarantee network robustness.

- To manage network lifetime and support event-driven mechanisms, network parameters need to be controlled and set up remotely and autonomously: the acquisition interval, the number of retransmissions allowed, the sensors to be activated, etc.

In this paper, we propose and analyze a WSN that adopts a synchronization procedure, a novel fault recovery (FR) protocol, and event-driven functionalities, all tailored for landslides monitoring. The aim of the developed WSN is to address most of the significant challenges of the monitoring scenario with available off-the-shelf communication technology. During the experimental campaign the network fulfilled most of the crucial requirements highlighted above. In particular: 1) The network operated for several months (almost 10) with negligible human intervention. The analysis of the residual battery charge after the experimental campaign is very encouraging, suggesting a network lifetime of more than one year in the worst case. 2) The WSN implements an eventdriven mechanism where continuous monitoring is performed with minimum energy resources and where augmented monitoring capabilities are triggered when relevant events occur. 3) Performance metrics and parameters such as radio links quality, path selection statistics, retransmission statistics and battery levels, are all available at the RU. In this way, the measurement campaign collected precious information on the network behavior which may help the network designer for future installations.

Our focus is on the description of the entire system and in analyzing its performance. The processing of geophysical data collected for landslide risk analysis is presented in [46].

The paper is organized as follows. Section II presents the scenario, the network deployment, and the sensors adopted for landslide monitoring. Synchronization, routing, fault tolerance capability and event-driven mechanisms implemented, are presented in Section III. Section IV describes the results of the extensive analysis of the network behavior based on performance metrics collected during the experimental campaign. Finally, Section V concludes the paper and summarizes the most important findings.

\section{Scenario Description And The Sensor Network}

The landslide considered for the test-bed is located in Torgiovannetto in a former quarry on the southward facing slope of Mount Subasio, $2 \mathrm{~km} \mathrm{NE}$ from the city of Assisi (Perugia, Umbria Region, Central Italy). It was first observed on May 2003 and it is assured that the main predisposing factor of the instability was the quarrying activity. The landslide, classified as a rockslide has a whole moving mass whose estimated volume is $182000 \mathrm{~m}^{3}$ [16], [46], [47]. ${ }^{1}$

\section{A. The sensors adopted}

The WSN designed for the Torgiovannetto landslide is composed of 15 wireless nodes, where one of these acts as network coordinator (NC). The nodes are equipped with 3 clinometers (tiltmeters), 4 wire extensometers, 2 bar extensometers, and 4 soil hygrometers. The NC acts as a gateway towards Internet through a general packet radio service (GPRS) modem to guarantee the access to the RU. The coordinator is also equipped with a weather station which includes several sensors: air thermometer, air hygrometer, rain gauge, wind gauge and soil hygrometer. A schematic view of the monitoring system is reported in Fig. 1, and a more detailed description of the network and the sensors is given in Table I.

\section{B. The sensor node}

The main components of a sensor node are a microcontroller, a transceiver, an external memory, a power source and one or more sensors [48]. The core of each node is the Texas Instrument CC2530 chip, which includes a $2.4 \mathrm{GHz}$ RF transceiver compliant with the IEEE 802.15.4 standard, and supports three low-power modes [49], [50].

The sensors adopted are of different types so their outputs require conditioning prior to the analog-to-digital conversion (ADC). In particular, the bar/wire extensometer and the air thermometer outputs are resistive, the tiltmeter and the hygrometer outputs are tensiometric, the anemometer and the rain gauge outputs are pulsed. Then, the corresponding digitized samples are stored locally into an $8 \mathrm{KByte}^{\text {EEPROM }^{2}}$ and inserted in the payload of the packet for transmission. The sensor node developed has a multi-layer structure to be customized on the monitoring needs (see Fig. 2).

As can be seen in Table I, every node is equipped with a sensor, except for the node with medium access control (MAC)

\footnotetext{
${ }^{1}$ Two main elements at risk are the nearby Provincial Road 249/1 and the Regional Road 444 . These roads are very important, since they are the only connection between the city of Assisi and the surrounding towns.

${ }^{2}$ The Microchip 24AA512 EEPROM supports more than $10^{6}$ erase/write cycles and data retention beyond 200 years. Considering one write-cycle every acquisition, at intervals $T_{\mathrm{ACQ}}=15 \mathrm{~min}$, the estimated EEPROM lifetime is around 29 years.
} 


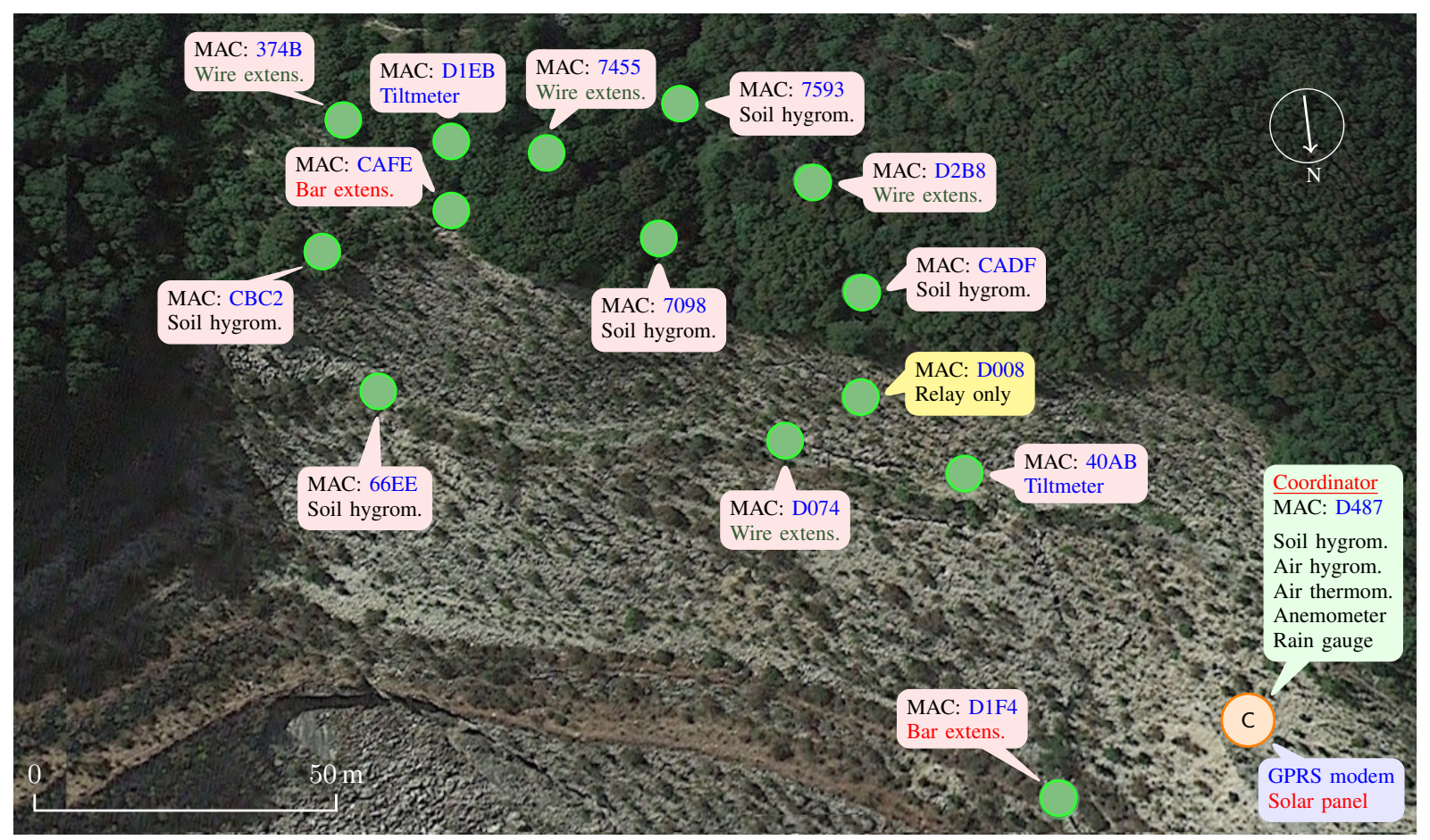

Fig. 1. The WSN installed on the Torgiovannetto (Central Italy) landslide.

TABLE I

THE NETWORK AND THE SENSORS

\begin{tabular}{|c|c|c|c|c|c|c|c|c|c|c|c|c|c|c|c|}
\hline MAC address $\rightarrow$ & D487 & D074 & D1F4 & $40 \mathrm{AB}$ & CADF & D2B8 & $66 \mathrm{EE}$ & CBC2 & $374 \mathrm{~B}$ & 7593 & CAFE & 7098 & D1EB & 7455 & D008 \\
\hline Logical address $\rightarrow$ & AABB & $x 74$ & $\mathrm{xF} 4$ & $\mathrm{xAB}$ & $\mathrm{xDF}$ & $\mathrm{xB} 8$ & $\mathrm{xEE}$ & $\mathrm{xC2}$ & $\mathrm{x} 4 \mathrm{~B}$ & $\mathrm{x} 93$ & $\mathrm{xFE}$ & $\mathrm{x} 98$ & $\mathrm{xEB}$ & $\times 55$ & $\mathrm{x} 08$ \\
\hline Latitude $\quad \mathrm{N} 43^{\circ} 4^{\prime}$ & $37.44 "$ & $35.72 "$ & $37.57^{\prime \prime}$ & $36.08^{\prime \prime}$ & $35.00^{\prime \prime}$ & 34.27 & $35.14^{\prime \prime}$ & $34.34 "$ & $33.53 "$ & $33.64 "$ & $34.15 "$ & $34.45^{\prime \prime}$ & $33.72^{\prime \prime}$ & $33.85 "$ & 35.58 \\
\hline Longitude E $12^{\circ} 38^{\prime}$ & $25.88 "$ & $28.95 "$ & $27.20^{\prime \prime}$ & $27.62^{\prime \prime}$ & 28.31" & $28.66^{\prime \prime}$ & $32.26 "$ & $32.86^{\prime \prime}$ & $32.80^{\prime \prime}$ & 29.78 & 31.77 & $29.98^{\prime \prime}$ & $31.80 "$ & 30.96 & 28.36 " \\
\hline Height a.s.l. & $551 \mathrm{~m}$ & $621 \mathrm{~m}$ & $590 \mathrm{~m}$ & $624 \mathrm{~m}$ & $659 \mathrm{~m}$ & $680 \mathrm{~m}$ & $625 \mathrm{~m}$ & $650 \mathrm{~m}$ & $668 \mathrm{~m}$ & $670 \mathrm{~m}$ & $658 \mathrm{~m}$ & $654 \mathrm{~m}$ & $679 \mathrm{~m}$ & $669 \mathrm{~m}$ & $652 \mathrm{~m}$ \\
\hline $\begin{array}{l}\text { Node type } \rightarrow \\
\text { Sensor type } \downarrow\end{array}$ & Coord. & \multicolumn{13}{|c|}{ Sensor } & Relay \\
\hline $\begin{array}{c}\text { Air hygrometer } \\
\text { range } 5 \div 95 \% \text {, acc. } \pm 2 \%\end{array}$ & $x$ & & & & & & & & & & & & & & \\
\hline $\begin{array}{c}\text { Air thermometer } \\
\text { range }-20 \div+80^{\circ} \mathrm{C} \text {, acc. } \pm 0.2 \%\end{array}$ & $x$ & & & & & & & & & & & & & & \\
\hline $\begin{array}{c}\text { Anemometer } \\
\text { speed acc. } \pm 5 \% \text {, dir. acc. } \pm 4^{\circ}\end{array}$ & $x$ & & & & & & & & & & & & & & \\
\hline $\begin{array}{l}\text { Rain gauge } \\
\text { res. } 0.2 \mathrm{~mm}\end{array}$ & $x$ & & & & & & & & & & & & & & \\
\hline $\begin{array}{c}\text { Wire extensometer } \\
\text { range } 250 \mathrm{~mm} \text {, acc. } 130 \mu \mathrm{m}\end{array}$ & & $x$ & & & & $x$ & & & $x$ & & & & & $x$ & \\
\hline $\begin{array}{c}\text { Bar extensometer } \\
\text { range } 150 \mathrm{~mm} \text {, acc. } 0.08 \mathrm{~mm}\end{array}$ & & & $\times$ & & & & & & & & $x$ & & & & \\
\hline $\begin{array}{c}\text { Tiltmeter } \\
\text { range } \pm 15^{\circ} \mathrm{C}, \text { acc. } \pm 0.02^{\circ}\end{array}$ & & & & $x$ & & & $x$ & & & & & & $x$ & & \\
\hline $\begin{array}{c}\text { Soil hygrometer } \\
\text { range } 5 \div 95 \% \text {, acc. } \pm 3 \%\end{array}$ & $x$ & & & & $x$ & & & $x$ & & $x$ & & $x$ & & & \\
\hline
\end{tabular}

address D008 which acts as a relay, and was included to increase network redundancy in a region with severe propagation impairments.

The wireless nodes have been insulated within a waterproof box with IP56 protection class, and installed on fixed tripods at approximately $1 \mathrm{~m}$ height.

\section{The batteries and the solar panel}

Sensor nodes are equipped with $6 \mathrm{~V} / 12 \mathrm{Ah}$ lead batteries, except for the $\mathrm{NC}$ which has a $6 \mathrm{~V} / 40 \mathrm{Ah}$ battery and a $30 \mathrm{~W}$ solar cell. The solar panel and the battery capacity have been chosen to satisfy the very high energy consumption of the
GPRS modem and considering that the landslide faces North with limited sun exposure.

\section{Remote management}

In the RU, data are saved on a MySQL database for further visualization, post-processing, and triggering of the eventdriven mode (see Section III-D). The RU is accessible through a web page with different sections:

- The Home page shows the most recent data collected with a time-stamp and the related MAC address. It is also possible to monitor network parameters such as the 


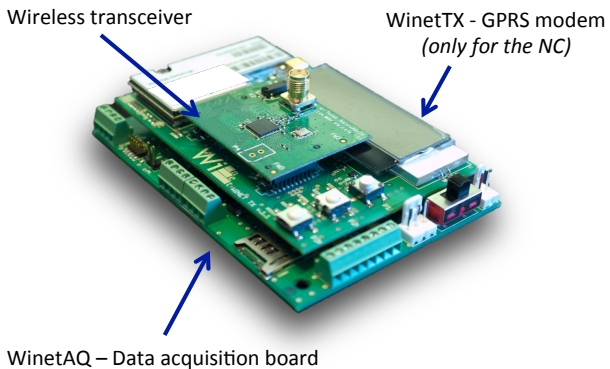

Fig. 2. The multi-layer architecture of the sensor node [48]

battery levels and the received signal strength (RSS) of radio links.

- The Plot section shows the time series of monitored parameters and of battery charge levels.

- The Map section shows the node positions on the landslide. The map is interactive and allows to visualize, e.g., the battery level of each node, the data collected by the sensor and the RSS. ${ }^{3}$

- The Warning section is a configuration page where it is possible to set up thresholds on monitored parameters and/or their rate of change, to detect an alarm situation. Such event, when occur, is followed by two actions:

1) an alarm message is sent (e.g., sms, e-mail, etc.);

2) the event-driven mechanism, as described in Section III-D, is activated.

- The Log section shows messages referred to network behavior, such as the activation of FR procedures, etc. These information are useful for network maintenance.

\section{Network Mechanisms FOR Robustness AND LONG LIFETIME IN HARSH ENVIRONMENTS}

The physical and MAC layer functionalities of each node are compliant with the IEEE 802.15.4 standard which allows a data rate of $250 \mathrm{Kbit} / \mathrm{s}$ [50].

As far as the network layer is concerned, we have developed an energy efficient ad-hoc protocol that implements mechanisms to ensure robustness and long lifetime through a self-organization protocol, a synchronization procedure, fault tolerant networking, and an event-driven network functionality.

Among different topologies and access mechanisms provided by the standard, we choose to exploit the peer-topeer mechanisms in non- Beacon-enabled mode. This setting allows the design of arbitrary topologies at network layer, e.g. trees [50], which better fit the monitoring scenario and sensor deployment. The MAC layer is an unslotted carrier sense multiple access/collision avoidance (CSMA/CA), which unfortunately is not suitable for our application. First, the CSMA/CA protocol aims at manage the access to the medium when node transmissions are not coordinated. This is in

\footnotetext{
${ }^{3}$ Each node is represented with a color which indicates the link quality: green for reliable communication (received power above $-80 \mathrm{dBm}$ ), orange for link with sufficient quality, red for critical link quality with frequent loss of packets (received power below $-90 \mathrm{dBm}$ ).
}

contrast to our monitoring system where data have to be collected at regular intervals (the acquisition time $T_{\mathrm{ACQ}}$ ) or at most at specific instants imposed by the RU. In applications such as this, it is much more efficient to organize the data gathering process in a coordinated manner. Second, as shown in Section IV-D, carrier sensing is a power consuming task as the energy spent in the receive phase (RP) by the wireless node is predominant. Therefore, performing carrier sensing for long time to allow uncoordinated access is prohibitive in a landslide scenario. Combining network mechanisms with a synchronization procedure, we designed a novel time division multiple access (TDMA) mechanism at network layer which exploits the peer-to-peer procedures made available by the standard. The advantage of this hybrid scheme, CSMA/CA at MAC layer and TDMA at network layer, in terms of energy savings, is two-fold:

- The new access scheme ensures very low probability of collision (alleviating the role of CSMA/CA at MAC level), with a significant saving in the energy spent for retransmissions.

- Synchronization among nodes leads to a significant increase of the sleep duration, $T_{\mathrm{SP}}$, with remarkable benefits for energy efficiency. For example, in our setting $T_{\mathrm{SP}} / T_{\mathrm{ACQ}}=99.6 \%($ see Table $\mathrm{V})$.

\section{A. Network self-organization}

Each node has a MAC address of 2 Bytes for data association and network management. The self-organizing protocol defines a tree logical network topology where each node has one father node and may have one or more child nodes, as depicted in Fig. 3. The protocol allows only communication toward nodes at a higher level with exception of the FR phase (see Section III-C).

To better reflect the network organization, the MAC address is paired with a logical address of the same length. In particular, the least significant byte of the logical address coincides with the one of the MAC address, while its most significant byte is the level to which the node belongs to, in the logical network topology. ${ }^{4}$ In Table I, nodes are identified by both addresses. The only exception is the NC, which is the sole root at level 0 , hence its logical address is simply AABB.

The self-organization protocol is driven by the association phase (AP) which establishes the logical topology of the network. In this phase, a node scans the radio channel to search a father node with good link quality. ${ }^{5}$ Then, if the father node is available, it creates a logical address and transmits it to the child node. ${ }^{6}$ If a node does not find any father, it enters into a FR phase.

\footnotetext{
${ }^{4}$ With this address mechanism the WSN can have up to 256 levels with 256 nodes each. The worst case scenario is represented by a network with linear topology with one node per level (a tree with only one branch), where the overall number of nodes is limited by the maximum number of levels, i.e., 256 .

${ }^{5}$ The threshold on the received power adopted to discriminate between good or bad link quality in the AP is $-80 \mathrm{dBm}$. This guarantees good network connectivity and prevents frequent FR procedures which are energy consuming.

${ }^{6} \mathrm{~A}$ candidate father could not be available because of low energy reserve or a failure.
} 


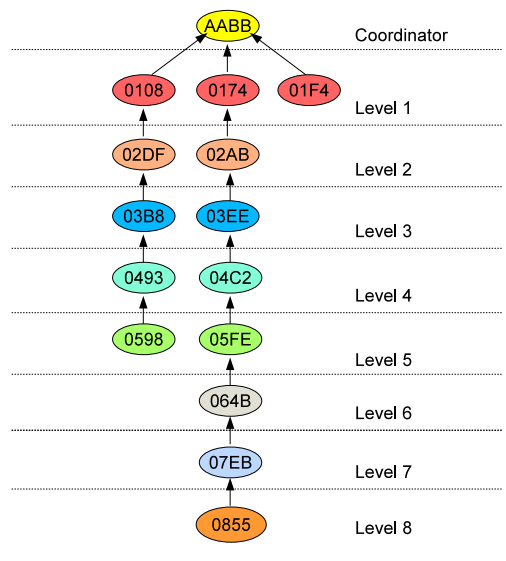

Fig. 3. The WSN tree logical topology.

Once the logical topology is completely formed, each node can be in one of four possible phases, which in normal conditions are visited cyclically:

- Association phase (AP). A node can accept node's association requests to become part of the network, or accept nodes that have been reset.

- Receive phase (RP). The node receives all the data which have been sent from child nodes, and stores them into the EEPROM in order of arrival.

- Transmit phase (TP). The node sends data collected from sensors first, then all neighbors' data according to a first-in-first-out (FIFO) policy, and finally wait for an ACK packet. If the transmission fails, data collected from sensors will be stored in the EEPROM until the communication towards the destination is restored. When the communication link is available again, the node sends its own historical data first, and then all neighbors' data.

- Sleep phase (SP). The radio interface is turned off, the CC2530 module enters into low-power mode 3, and neither transmission nor reception are possible.

Each phase corresponds to a specific temporal slot. In particular: the association slot has duration $T_{\mathrm{AP}}=1500 \mathrm{~ms}$; the receiving slot has a variable duration, $T_{\mathrm{RP}}$, which depends on the amount of data transmitted by child nodes, with a minimum value of $T_{\mathrm{RP}, \min }=2100 \mathrm{~ms}^{7}{ }^{7}$ the data transmission slot, $T_{\mathrm{TP}}$, is also varied dynamically based on the volume of data to be passed to the father node; for the rest of the time nodes are in SP with duration $T_{\mathrm{SP}}$. The acquisition interval

$$
T_{\mathrm{ACQ}}=T_{\mathrm{AP}}+T_{\mathrm{RP}}+T_{\mathrm{TP}}+T_{\mathrm{SP}}
$$

has a fixed duration and can be set remotely on the RU. Note that $T_{\mathrm{ACQ}}$ is the time interval between two consecutive data acquisitions and is fetched by the NC during each GPRS connection activated for data transfer. To disseminate such information to the whole network, there is a dedicated time slot where all nodes wake up simultaneously and exchange

\footnotetext{
${ }^{7}$ To keep energy consumption under control, if a node does not receive any data within $T_{\mathrm{RP}, \min }$, it will be forced to switch to data transmission and then pass into the SP.
}

$T_{\mathrm{ACQ}}$ in broadcast. ${ }^{8}$

Note that, if the node positions and propagation conditions are favorable, the self-organizing procedure may choose a star topology which ensures maximum energy saving due to single hop communications toward the NC.

\section{B. Network synchronization}

Network synchronization guarantees the alignment of temporal slots of different phases between nodes [51]. In particular, the alignment ensures that whenever a child node enters in a TP, the father node is in the RP. To this aim, the nodes have different wake up times from the SP based on the logical level they belong to. To illustrate this procedure, Fig. 4 shows how the active time slots of three nodes, which belong to different levels, are synchronized. Note that the synchronization procedure aims at differentiate the wake up times of the nodes to control the access to the medium in a TDMAlike fashion. Solutions like the flooding time-synchronization protocol [52] may ensure a tight synchronization at the cost of signaling packets dedicated to timing preservation. As tight synchronization is not mandatory in our monitoring scenario, we developed a synchronization procedure whose aim is to trade off between signaling overhead and timing accuracy.

Synchronization is based on a real-time clock (RTC) provided on each node. The clock chip used is the STMicroelectronics M41T81S which has a drift in the range $[-1.1,+1.9]$ minute/month when the temperature is in the range $[-5,+55]^{\circ} \mathrm{C}$. With these features it is possible to maintain nodes in sleep state for a very long time without harmful misalignments. ${ }^{9}$ The start up phase of the network begins turning on the $\mathrm{NC}$ which fetches date and time from the RU through the GPRS connection. These information are used to set the NC clock and are propagated through the network during the AP of each node, so as to guarantee updated time references. In particular, after completing the association and then receiving the logical address from the father node, the child reads the sensors, transmits all the collected data, and wait for an ACK packet containing updated time and date.

As presented in Section III-C, because of the FR mechanism it is possible that a node changes the level while keeping its short address to avoid a new association. Therefore, there is no direct link between a node level and a suitable wake up time to allow a synchronization with the upper level nodes. For this reason, to ensure the temporal alignment with the child node, every father node sends its wake up time within the ACK packet.

\section{Fault recovery $(F R)$ procedure}

The fault tolerance mechanism is the management of events which cause nodes isolation due to e.g., low radio link quality with all neighbors, a failure, or insufficient battery charge. Because of the harsh operating environment, the very long network lifetime, and the lack of human intervention, these

\footnotetext{
${ }^{8}$ Such time slot is very short, so it does not affect the energy consumption significantly.

${ }^{9}$ In the network setting we consider $T_{\mathrm{ACQ}} \leq 15 \mathrm{~min}$, but longer acquisition intervals are possible.
} 


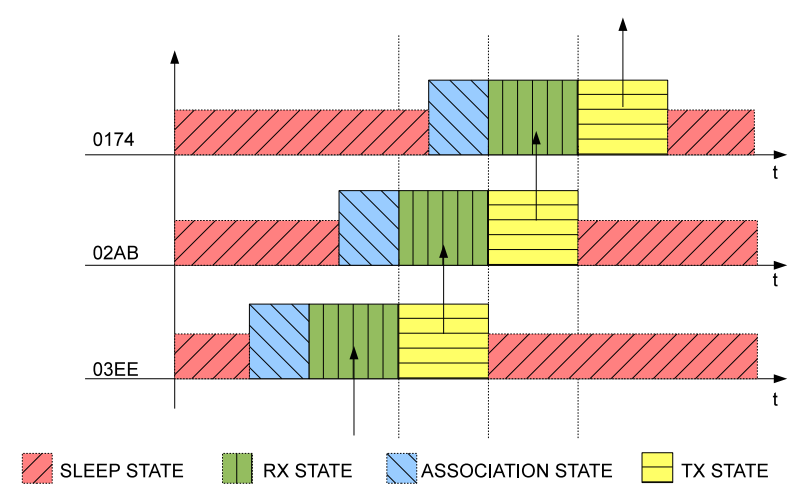

Fig. 4. Time synchronization among three nodes belonging to different levels.

situations may occur unexpectedly. The novel FR procedure designed is based on the following criteria:

I) In the TP, a node has knowledge of the correct data delivery when it receives an ACK packet. Every time a node does not receive the ACK, it keeps all the data in its memory to deliver them during the following awakening. If the ACK is not delivered for two consecutive times, the link is declared unreliable and the node must look for a new father to communicate with.

II) A node search for a new father among the ones on an upper level.

III) To search for a new father it is not necessary to start a new AP. Instead, a special packet containing the following information is sent in broadcast mode:

- the level, $L_{\mathrm{f}}$, the new father node must belong to;

- the wake up time, $T_{\mathrm{f}}$, of the new father node.

During the first search, $L_{\mathrm{f}}$ is simply the level right above the one the node belongs to. In case there are no fathers available in such level, the search continues to a higher level. Following such mechanism, a node is able to rise the network hierarchy, if needed, till level 1. According to the synchronization mechanism described in Section III-B if a node rises up from one level to another, it must update its wake up time to be synchronous with the new father.

IV) If III) fails, i.e., after reaching level 1 a communication towards the NC is still not possible, the node becomes orphan and goes back to its initial level to start again a new search and, if necessary, climb again the hierarchy. As soon as it becomes orphan, the node sets its sleep interval, $T_{\mathrm{SP}}$, to 1 minute, irrespective of its previous value. This action, forces the orphan to have short sleep duration to quickly recover the synchronization with a candidate father. In fact, an orphan could have been isolated from the network for several minutes and could have missed the packet containing an updated $T_{\mathrm{ACQ}}$ fetched from the RU, or can be subject to a temporal drift. Once the orphan has found a father node, its sleep time is restored according to the updated $T_{\mathrm{ACQ}}$.

The second parameter, $T_{\mathrm{f}}$, guarantees that a father node belonging to a superior level with the correct synchronism is found.

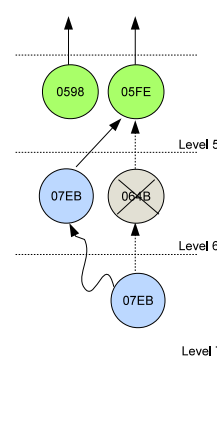

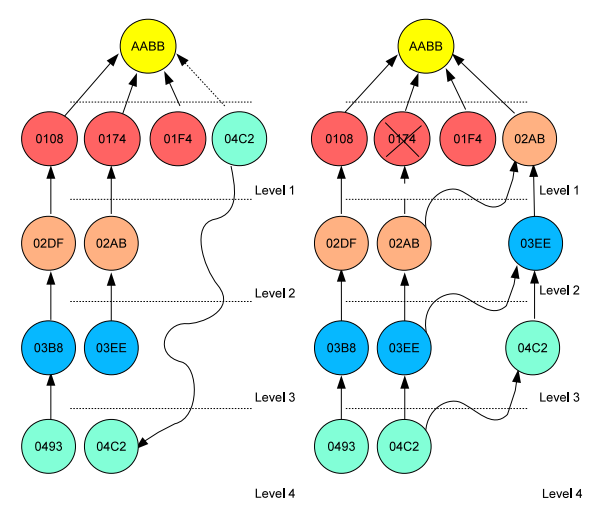

c
Fig. 5. Fault recovery mechanisms: a) level migration; b) go back to the initial level; c) group level migration.

V) An orphan never replies to its child with an ACK. This criterion force child nodes to look for another father to avoid isolation from the rest of the network.

To better illustrate the FR procedure, Fig. 5 depicts three possible situations: a) the migration of a single node to an upper level; b) a node going back to the initial level after becoming orphan; c) the migration of a group of nodes. In particular, in Fig. 5a, node 07EB after transmitting twice its data to node 064B without receiving any ACK, starts looking for another father at level 6 , but since it cannot find it, it migrates to the upper level. Fig. 5b shows node 04C2 which cannot communicate anymore with its father 03EE. Hence, the node starts migrating from level to level until reaching level 1 , but since the transmission toward the $\mathrm{NC}$ fails, it becomes orphan and goes back to level 4 , where it starts a new search. Finally, in Fig. 5c the node $02 \mathrm{AB}$ cannot communicate any longer with its father 0174, and since it does not find other fathers at level 1 , it migrates to the upper level to communicate with the NC. Since this node gets data from child node 03EE, which in turn is node 04C2's father, even these last two nodes migrate to upper levels.

The FR mode ensures that data collected and stored in the EEPROM is delivered to the NC using the most favorable path. Data collected are lost only in the case the FR procedure fails consecutively a number of times such that the samples received from the sensors saturate the memory of the node.

\section{Event-driven network control}

The event-driven mechanism is based on the analysis of data collected and stored on the RU.

Within the configuration page of the RU it is possible to set up specific thresholds for each sensor, to activate the alarm and the event-driven mode (EDM), based on the status of the landslide. The event-driven mechanism autonomously increases the data acquisition rate, so as to quickly react to a critical 
TABLE II

FRACTION OF PACKETS SENT (FPS) BETWEEN NODES IN THE WSN

\begin{tabular}{|c|c|c|c|c|c|c|c|c|c|c|c|c|c|c|c|c|}
\hline $\begin{array}{c}\mathcal{R} \rightarrow \\
\mathcal{T} \downarrow\end{array}$ & 0108 & 0174 & $01 \mathrm{~F} 4$ & $02 \mathrm{AB}$ & $02 \mathrm{DF}$ & 03B8 & 03EЕ & $03 \mathrm{C} 2$ & 044B & 0493 & $05 \mathrm{FE}$ & 0598 & 05EB & 0655 & $\begin{array}{l}\text { AABB } \\
\text { Coord. }\end{array}$ & $\begin{array}{l}\text { Fault Rec. } \\
\text { Mode }\end{array}$ \\
\hline 0108 & & & & & & & & & & & & & & & $93.61 \%$ & $6.39 \%$ \\
\hline 0174 & & & & & & & & & & & & & & & $92.19 \%$ & $7.81 \%$ \\
\hline $01 \mathrm{~F} 4$ & $1.09 \%$ & $10.23 \%$ & & & & & & & & & & & & & $83.01 \%$ & $5.67 \%$ \\
\hline $02 \mathrm{AB}$ & $21.17 \%$ & $36.43 \%$ & $14.3 \%$ & & & & & & & & & & & & $19.44 \%$ & $8.66 \%$ \\
\hline $02 \mathrm{DF}$ & $87.63 \%$ & $5.35 \%$ & $0.54 \%$ & & & & & & & & & & & & $0.74 \%$ & $5.74 \%$ \\
\hline 03B8 & $9.64 \%$ & & & & $82.51 \%$ & & & & & & & & & & $4.27 \%$ & $3.58 \%$ \\
\hline 03EE & $10.6 \%$ & $10.17 \%$ & & $24.82 \%$ & $0.79 \%$ & & & & & & & & & & $45.95 \%$ & $7.67 \%$ \\
\hline $03 \mathrm{C} 2$ & & & $1.47 \%$ & $6.48 \%$ & $1.55 \%$ & & $15.7 \%$ & & & & & & & & $0.41 \%$ & $74.39 \%$ \\
\hline 044B & & $14.02 \%$ & & $2.06 \%$ & & & $3.12 \%$ & $9.25 \%$ & & & $18.11 \%$ & & & & $33.77 \%$ & $19.67 \%$ \\
\hline 0493 & $2.29 \%$ & & & & $8.84 \%$ & $85.46 \%$ & & & & & & & & & & $3.41 \%$ \\
\hline $05 \mathrm{FE}$ & & & $5.97 \%$ & $14.67 \%$ & & $0.09 \%$ & $45.25 \%$ & $16.09 \%$ & $3.24 \%$ & $7.04 \%$ & & & & & $2.12 \%$ & $5.53 \%$ \\
\hline 0598 & $2.35 \%$ & $16.66 \%$ & $13.58 \%$ & $17.1 \%$ & $8.17 \%$ & $3.68 \%$ & $0.79 \%$ & & & $15.97 \%$ & & & & & $16.89 \%$ & $4.81 \%$ \\
\hline $05 \mathrm{~EB}$ & & & & & $2.61 \%$ & $7.4 \%$ & $0.03 \%$ & $2.72 \%$ & $43.06 \%$ & $34 \%$ & & & & & & $10.18 \%$ \\
\hline 0655 & $0.69 \%$ & & & & $0.2 \%$ & $22.68 \%$ & & & $2.21 \%$ & $4.68 \%$ & $32.49 \%$ & $10.41 \%$ & $22.47 \%$ & & $0.41 \%$ & $3.76 \%$ \\
\hline
\end{tabular}

situation and allow augmented monitoring capabilities. ${ }^{10}$ The interval, $T_{\mathrm{ACQ}}$, once modified by the event-driven mechanism, is disseminated by the $\mathrm{NC}$ in broadcast mode through the dedicated time slot.

The EDM has a duration, $T_{\mathrm{EDM}}$, which can be selected by the user or can be autonomously determined by the system based on the classification of the type of event occurred. Note that both $T_{\mathrm{ACQ}}$ and $T_{\mathrm{EDM}}$ determine the energy consumption in the EDM; in fact a reduction of $T_{\mathrm{ACQ}}$ corresponds to a shorter SP. These parameters have to be carefully chosen for a reasonable trade-off between network lifetime and augmented monitoring capabilities.

\section{Sensor Network Statistics During the MONitoring CAMPAigN}

In this section we present some network statistics extracted by the analysis of data collected during the monitoring campaign from February to October 2013.

The volume of information collected and delivered during an acquisition can be estimated considering that each node send 17 Byte of data except the relay node which send 15 Byte. Therefore, the 13 nodes plus the relay collected and delivered to the NC approximately $13 \times 17$ Byte +15 Byte $=236$ Byte every $T_{\mathrm{ACQ}}=15 \mathrm{~min}$.

\section{A. Paths statistics}

An important metric to understand the behavior of the routing algorithm is link utilization. More precisely, for a link identified by a transmitting node, $\mathcal{T}$, and a receiving node, $\mathcal{R}$, we define the fraction of packets sent (FPS) as

$$
\operatorname{FPS}(\mathcal{T}, \mathcal{R})=\frac{\# \text { of packets sent from } \mathcal{T} \text { to } \mathcal{R}}{\text { total \# of packets sent by } \mathcal{T}}
$$

Such metric is reported (in percentage) in Table II for all the links established in the WSN. The FPS includes also the number of packets sent during the FR procedure to look for

\footnotetext{
${ }^{10}$ During the monitoring campaign in Torgiovennetto we chose $T_{\mathrm{ACQ}}=$ $15 \mathrm{~min}$ as default value for normal operations in the absence of alarms. During a critical situation detected by the RU the system can reduce the acquisition interval up to $T_{\mathrm{ACQ}}=1 \mathrm{~min}$ in the $\mathrm{EDM}$, if needed.
}

a father node (last column). Hence, since each node can have only one father, the sum of values in each row is equal to $100 \%$.

As can be seen in the table, each node transmits data only to nodes belonging to a higher level. Therefore, node 0108 from level 1 delivers all its data to the NC, except for a certain percentage of transmissions it was in FR mode. Nodes 01F4, $03 \mathrm{C} 2$ and $044 \mathrm{~B}$ show some exceptions to the above rule. In fact, node 01F4 experienced an unreliable radio link with node 0174, which belongs to the same level, while node 044B has had difficulties to communicate with 05FE, which stands on a lower level. Such exceptions are due to nodes reset (caused by an electrostatic discharge during a thunderstorm) which brought to a re-association of the nodes. As a consequence, the logical addresses shown in the table are the one after the reset, since previously these nodes belonged to a lower level. The node $03 \mathrm{C} 2$ experienced a similar critical situation, confirmed by the highest FRs rate among all nodes. In fact, Table II shows that a favorite link is toward 03EE before a reset, and then toward $02 \mathrm{AB}$, which unfortunately is quite far (see Fig. 1 and Fig. 6). Therefore, $03 \mathrm{C} 2$ has found difficulties while trying to deliver data to the NC. This situation had a remarkable impact on energy consumption of this node as will be detailed in Section IV-D.

Fig. 6 depicts Table II data referred to the transmitting node 0655, where the color represents the level to which the receiver belongs to. The figure reveals that such node established most of data transmissions towards nodes $05 \mathrm{~EB}$ and $05 \mathrm{FE}$, and in some cases it even migrated to level 4 to communicate with node 03B8.

A different analysis is offered by Fig. 7 which shows the most used paths towards the NC (we considered only links with a FPS greater than 15\%). For example, data gathered by node 0493 is able to reach the coordinator by hopping through nodes 03B8, 02DF, and 0108. In the same figure we can see how some nodes, for instance nodes 0598 and $02 \mathrm{AB}$, have 
different preferred paths. ${ }^{11}$

\section{B. Packet statistics}

A metric that quantifies the link quality at network level is the packet retransmission rate (PRR) between two nodes, $\mathcal{T}$ and $\mathcal{R}$, defined as

$$
\operatorname{PRR}(\mathcal{T}, \mathcal{R})=\frac{\# \text { of packets retransmitted from } \mathcal{T} \text { to } \mathcal{R}}{\text { total \# of packets sent by } \mathcal{T}} .
$$

Table III shows the PRR in percentage for each link in the WSN. The PRR values can be related to data provided in Table II, since links with a high PRR are, in general, the less used. There are of course the effects of node associations, battery levels and also temporal fluctuations of radio channels, that may cause a non direct correspondence between PRR and FPS. For example, node $02 \mathrm{AB}$ communicates with node 0174 for $36.43 \%$ of times, and with node 0108 for $21.17 \%$ of times, but the first link has a PRR slightly higher than the second one.

An interesting aspect is related to the relay-only node 0108 , added to the WSN to ensure good connectivity in the region close to the NC. In fact, because of the physical topology of the WSN and the radio propagation along the landslide, a lack of connectivity in this part of the network may cause difficulty in delivering all collected data toward the NC. In fact, looking at Fig. 7 such relay is responsible for delivering most of the data collected in the upper side of the WSN, i.e., nodes 0493 , 03B8, and 02DF.

\section{Radio link statistics}

From the radio propagation point of view, the RSS is the most used and easy to measure parameter to quantify the link quality [53]. In Table IV, the mean value and the standard deviation of the RSS for all links in the network are reported. As a reference, the receiver sensitivity of CC2530 is $-97 \mathrm{dBm}$. As can be seen, the standard deviation of the RSS ranges from $0.7 \mathrm{~dB}$ to $4.9 \mathrm{~dB}$. Such values can be used to set up a proper fading margin for future installations of the WSN in similar environments.

Based on Table IV, it is also interesting to analyze the behavior of links to better understand the joint impact of propagation and protocol aspects to the formation of the network. As can be noted, the link between the node 0174 and the NC has a rather high mean RSS, $-66.5 \mathrm{dBm}$, with low standard deviation, $0.9 \mathrm{~dB}$, compared to that of nodes 0108 and 01F4. Observing the position of these nodes in Fig. 6, it is clear that such difference is not due to the distance, since node $01 \mathrm{~F} 4$ is closer to the NC compared to node 0174 , and neither by the presence of trees and foliage, since these

\footnotetext{
${ }^{11}$ Path changes depends on several factors. The two most important are temporal variations in signal propagation and battery consumptions. The first aspect is related to the fact that radio propagation in a landslide environment is only apparently static, because in a real environment the foliage has a density which follows seasonal changes. Regarding the second aspect, the non-uniform battery consumption among nodes, which depends on the type of sensor the node is connected to and on the protocol behavior, has an effect on the paths selected.
}

nodes are placed outside the forest. Rather, the difference lies in the fact that nodes are positioned at different altitudes, hence the irregular surface of the landslide is the main cause of obstructions for some links. Regarding the node 02DF, it established weak radio links towards the nearest nodes (RSS less than $-90 \mathrm{dBm}$ ), except with the closest one, node 0108 (see Fig. 1). As expected, Table II and Table III confirm that such link is the mostly used and the one with the lowest PRR. As far as node 0598 is concerned, it belongs to level 5 and is placed in a central position, hence it is the node which communicated with the highest number of nodes, 9 in particular, and experienced a quite low FR rate. This confirms that a dense network with good connectivity can reduce FR procedures and guarantees higher lifetime.

\section{Energy consumption and network lifetime}

In Table $\mathrm{V}$, the power and energy consumption of the wireless node during an acquisition interval in the TP, RP and $\mathrm{SP}$, are reported. To derive the energy consumption we provide the current absorbed by the radio transceiver, the one supplied to the whole node (considering a reference voltage supply of $6.4 \mathrm{~V}$ ) and the duration of each phase. We do not consider the AP which occurs at the beginning during network formation and only occasionally during the FR procedure. To increase energy saving, the SP adopts the Power Mode 3, which, among the three available in the CC2530 chip, is the one with the lowest current. As can be seen, in both TP and RP the overall consumption is dominated by the radio transceiver with $1 \mathrm{~mA}$ extra current supplied to the data acquisition board. In the SP the overall current absorbed of the wireless node is quite low, $30 \mu \mathrm{A}$, and is due in part to the presence of the RTC for network synchronization by timed wake-ups. As can be noticed the RP is the most energy demanding $(76.92 \%)$ mostly because of its duration, more than three order of magnitude with respect to the TP. Thanks to the adoption of a network synchronization and a TDMA, such duration, $T_{\mathrm{RP}}$ have been kept enough small to save energy but sufficiently large to accommodate multiple receptions from neighbor nodes, allow network synchronization, and support the FR procedure.

In Table VI we provide the current, voltage, and energy consumption of the sensors attached to the nodes. To better understand the impact of sensor on the overall energy consumption, the last column shows also the ratio between the energy consumption of the sensor and that of the node. The energy spent by the sensor has been calculated on a time interval of $2100 \mathrm{~ms}$, during which the sensor is turned on, while the overall energy spent by the node is obtained from Table V.

To complete the analysis of the network behavior and to estimate its lifetime, we collected battery voltages corresponding to each node, during the measurement campaign. Such values are reported in Fig. 8. ${ }^{12}$ Note that the unequal initial values are due to different battery charge levels and also to the fact that the network was already active before the measurement campaign. As reference levels, a battery is considered fully

\footnotetext{
${ }^{12}$ To avoid many overlapped curves, the figure shows battery voltages for a subset of the nodes.
} 




Fig. 6. Radio links established by node 0655. The labels show the FPS as reported in Table II.

TABLE III

PACKET RETRANSMISSION RATE (PRR) FOR EACH RADIO LINK IN THE WSN

\begin{tabular}{|c|c|c|c|c|c|c|c|c|c|c|c|c|c|c|c|}
\hline $\begin{array}{c}\mathcal{R} \rightarrow \\
\mathcal{T} \downarrow\end{array}$ & 0108 & 0174 & $01 \mathrm{~F} 4$ & $02 \mathrm{AB}$ & $02 \mathrm{DF}$ & 03B8 & 03EE & $03 \mathrm{C} 2$ & 044B & 0493 & $05 \mathrm{FE}$ & 0598 & 05EB & 0655 & $\begin{array}{l}\text { AABB } \\
\text { Coord. }\end{array}$ \\
\hline 0108 & & & & & & & & & & & & & & & $6.37 \%$ \\
\hline 0174 & & & & & & & & & & & & & & & $7.79 \%$ \\
\hline $01 \mathrm{~F} 4$ & $13.79 \%$ & $4.59 \%$ & & & & & & & & & & & & & $5.08 \%$ \\
\hline $02 \mathrm{AB}$ & $2.8 \%$ & $5.65 \%$ & $3.44 \%$ & & & & & & & & & & & & $15.35 \%$ \\
\hline $02 \mathrm{DF}$ & $2.49 \%$ & $11.96 \%$ & $32.73 \%$ & & & & & & & & & & & & $61.36 \%$ \\
\hline 03B8 & $4.78 \%$ & & & & $1.38 \%$ & & & & & & & & & & $20.44 \%$ \\
\hline 03EE & $3.41 \%$ & $2.08 \%$ & & $1.88 \%$ & $11.29 \%$ & & & & & & & & & & $9.26 \%$ \\
\hline $03 \mathrm{C} 2$ & & & $4.17 \%$ & $12.79 \%$ & $3.97 \%$ & & $1.21 \%$ & & & & & & & & $93.59 \%$ \\
\hline 044B & & $10.82 \%$ & & $10.56 \%$ & & & $22.42 \%$ & $2.36 \%$ & & & $1.17 \%$ & & & & $13.13 \%$ \\
\hline 0493 & $6.55 \%$ & & & & $3.19 \%$ & $1.23 \%$ & & & & & & & & & \\
\hline $05 \mathrm{FE}$ & & & $3.08 \%$ & $1.08 \%$ & & $40 \%$ & $3.03 \%$ & $0.9 \%$ & $6.72 \%$ & $8.54 \%$ & & & & & $12.12 \%$ \\
\hline 0598 & $11.6 \%$ & $2.49 \%$ & $3.46 \%$ & $0.85 \%$ & $4.46 \%$ & $7.04 \%$ & $1.82 \%$ & & & $2.33 \%$ & & & & & $1.37 \%$ \\
\hline 05EB & & & & & $17.33 \%$ & $7.78 \%$ & $66.67 \%$ & $16.35 \%$ & $3.76 \%$ & $1.27 \%$ & & & & & \\
\hline 0655 & $7.84 \%$ & & & & $30 \%$ & $0.39 \%$ & & & $3.21 \%$ & $1.84 \%$ & $2.88 \%$ & $1.39 \%$ & $1.16 \%$ & & $26.32 \%$ \\
\hline
\end{tabular}

charged when its voltage is above $V_{\mathrm{C}}=6.4 \mathrm{~V}$, and discharged when its voltage goes below $V_{\mathrm{D}}=6.2 \mathrm{~V} \cdot{ }^{13}$

As can be noted in Fig. 8, all nodes have been always active with enough battery charge, except node $03 \mathrm{C} 2$. Its lifetime was around 3.5 months with a rather fast battery discharge starting from end of March up to June. Such depletion is explained by the very high FR rate, $74.39 \%$, as discussed in Section IV-A. As can be seen, the battery has been fully recharged in mid July. Another critical situation involves node 05EB which did not required any battery recharge but reached the minimum voltage, $V_{\mathrm{D}}$, at the end of the measurement campaign. Similarly to node $03 \mathrm{C} 2$, this node experienced

\footnotetext{
${ }^{13}$ Such value is quite conservative. Actually, depending on the hardware and sensors installed on the nodes, $V_{\mathrm{D}}$ can be even lower, guaranteeing longer lifetimes.
}

frequent FRs, but this is not enough to justify the battery depletion since, e.g., node 044B had higher FR rates but with a much slower discharge. The reason has to be found in Table III, where we can notice that node 05EB has had a very high PRR toward node 03EE.

In our scenario, based on the characteristics of the landslide (it is a rockslide with very slow variation of the parameters) and the monitoring needs, we choose to define the network lifetime as the time duration from the deployment of the WSN to when a fraction $\delta$ of the $N=15$ nodes run out of energy. More precisely, considering the ordered set of node lifetimes $t_{1} \leq t_{2} \leq \ldots \leq t_{N}$, then

$$
T_{\text {life }}=t_{k} \quad \text { with } \quad k=\lceil\delta \cdot N\rceil
$$

where $\lceil x\rceil$ stands for the smallest integer not less than $x$. During the measurement campaign we collected the node lifetimes 


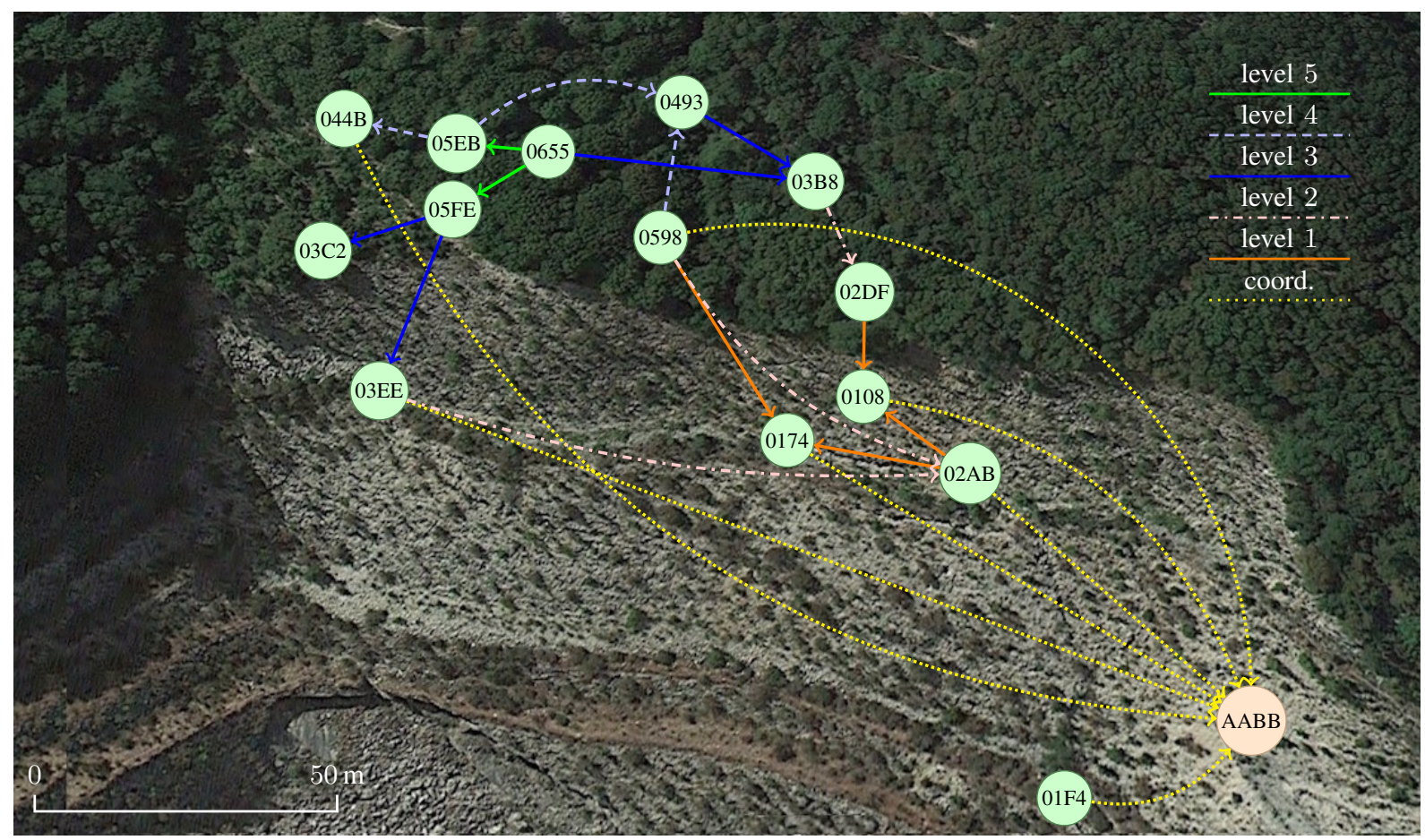

Fig. 7. Most used links, with FPS $>15 \%$, during the monitoring campaign.

TABLE IV

MEAN VALUE IN $d B m$ (STANDARD DEVIATION IN $d B$ ) OF RSS FOR EACH RADIO LINK

\begin{tabular}{|c|c|c|c|c|c|c|c|c|c|c|c|c|c|c|}
\hline$\frac{\mathcal{R} \rightarrow}{\mathcal{T} \downarrow}$ & 0108 & 0174 & $01 \mathrm{~F} 4$ & $02 \mathrm{AB}$ & $02 \mathrm{DF}$ & 03B8 & 03EЕ & $03 \mathrm{C} 2$ & 044B & 0493 & $05 \mathrm{FE}$ & 0598 & 05EB & $\begin{array}{l}\text { AABB } \\
\text { Coord. }\end{array}$ \\
\hline 0108 & & & & & & & & & & & & & & $-78(2.6)$ \\
\hline 0174 & & & & & & & & & & & & & & $-66.5(0.9)$ \\
\hline $01 F 4$ & $-86.1(1.1)$ & \begin{tabular}{|l|}
$-81.3(1.5)$ \\
\end{tabular} & & & & & & & & & & & & $-76.3(2.0)$ \\
\hline $02 \mathrm{AB}$ & $-81.9(2.6)$ & $-67.4(2.7)$ & $-87.1(1.6)$ & & & & & & & & & & & $-71.2(0.9)$ \\
\hline $02 \mathrm{DF}$ & $\begin{array}{l}-75.5(4.9) \\
\end{array}$ & \begin{tabular}{|l|}
$-92.8(2.0)$ \\
\end{tabular} & $-97.8(1.1)$ & & & & & & & & & & & $-90.8(3.0)$ \\
\hline $03 \mathrm{~B} 8$ & \begin{tabular}{|l|}
$-79.7(3.7)$ \\
\end{tabular} & & & & $-62.8(1.6)$ & & & & & & & & & $-95.5(1.0)$ \\
\hline 03EE & $-83.5(1.6)$ & \begin{tabular}{|l|}
$-78.7(1.5)$ \\
\end{tabular} & & $-77.7(2.0)$ & $-93.1(3.5)$ & & & & & & & & & $-80.9(2.3)$ \\
\hline $03 \mathrm{C} 2$ & & & $-86.6(1.1)$ & $-74.1(1.6)$ & $-93.7(1.2)$ & & \begin{tabular}{|l|}
$-77.1(1.9)$ \\
\end{tabular} & & & & & & & $-75.2(1.7)$ \\
\hline 044B & & \begin{tabular}{|l|}
$-94.5(1.9)$ \\
\end{tabular} & & $-95.3(1.2)$ & & & \begin{tabular}{|l|}
$-97.2(1.2)$ \\
\end{tabular} & $-71.8(1.9)$ & & & $-60.9(1.8)$ & & & $-94.3(1.4)$ \\
\hline 0493 & $-93.2(1.3)$ & & & & $-66.8(1.6)$ & $-77.8(4.4)$ & & & & & & & & \\
\hline $05 \mathrm{FE}$ & & & $-92.5(1.3)$ & $-89(1.5)$ & & $-96(1.4)$ & $-85.2(3.0)$ & $\begin{array}{l}-76.4(3.9) \\
\end{array}$ & $-63(1.4)$ & $-97.4(1.1)$ & & & & $-87.1(1.3)$ \\
\hline 0598 & $-88.2(3.9)$ & $-70.3(1.6)$ & $-90.6(2.6)$ & $-82.2(2.6)$ & $-84.7(4.5)$ & $-88.2(2.9)$ & $-95.6(1.1)$ & & & $-88.2(4.1)$ & & & & $-74.1(2.4)$ \\
\hline $05 \mathrm{~EB}$ & & & & & $-95.2(2.0)$ & $-94.1(2.1)$ & $-98.5(0.7)$ & $-94.7(2.1)$ & $-69.7(1.4)$ & $-91.8(2.9)$ & & & & \\
\hline 0655 & $-96.4(1.1)$ & & & & $-93.7(3)$ & $-84.4(3.3)$ & & & $-92.6(1.5)$ & $-85.3(4.6)$ & $-88.2(4.6)$ & $-90.1(2)$ & $-71.4(2.6)$ & $-97.9(0.6)$ \\
\hline
\end{tabular}

$t_{1}=121$ days for node $03 \mathrm{C} 2$, and $t_{2}=242$ days for node 05EB, as can be seen in Fig. 8. Fixing a fraction of the number of nodes allowed to run out of energy equal to $\delta=10 \%$, we have $k=\lceil 0.1 \cdot 15\rceil=2$, thus $T_{\text {life }}=t_{2}=242$ days, i.e., beyond 9 months. For the rest of the network, at the end of the monitoring period, node batteries voltage was above $6.3 \mathrm{~V}$, confirming that node lifetimes can be safely estimated well beyond one year.

Regarding the NC, despite the landslide is oriented to North, with a reduced sun exposure, especially in winter, the solar panel provided enough power to reach a complete charge. Considering such good behavior, the coordinator lifetime is only limited by battery degradation, hence of several years.

\section{CONCLUSION}

This paper presented a WSN designed for landslides supervision, by monitoring several parameters, to assess the possible risks and provide useful information for an early warning system. All data recorded are collected and organized into a data-base for data logging and post processing on a remote server. To ensure energy efficiency and reliable communications in harsh environments, we developed a new network protocol and we designed dedicated hardware for the sensor nodes. Moreover, to have full control of energy consumption, network lifetime, and required data acquisition rate, all relevant parameters can be set up remotely on-the-fly.

The network operated on a landslide for several months with negligible human intervention. During the monitoring campaign several performance metrics such as radio link quality, packets transmission statistics, path selection statistics, and 
TABLE V

ENERGY AND POWER CONSUMPTION OF THE WIRELESS NODE DURING AN ACQUISITION INTERVAL $\left(T_{\mathrm{ACQ}}=15\right.$ MIN $)$

\begin{tabular}{|c|c|c|c|c|c|c|c|c|}
\hline \multirow{2}{*}{$\begin{array}{c}\text { State } \downarrow \\
\text { Transmit phase (TP) } \\
1 \mathrm{dBm} \text { TX power }\end{array}$} & \multirow{2}{*}{$\begin{array}{l}\text { Current } \\
C C 2530\end{array}$} & \multirow{2}{*}{$\begin{array}{c}\text { Current } \\
\text { node }\end{array}$} & \multicolumn{2}{|c|}{$\begin{array}{c}\text { Power [percentage] } \\
\text { node }\end{array}$} & \multicolumn{2}{|c|}{ Duration [percentage] } & \multicolumn{2}{|c|}{$\begin{array}{c}\text { Energy [percentage] } \\
\text { node }\end{array}$} \\
\hline & & & $192 \mathrm{~mW}$ & $54.51 \%$ & $2.56 \mathrm{~ms}^{a}$ & $0.00028 \%$ & $0.000492 \mathrm{~J}$ & $0.0658 \%$ \\
\hline Receive phase (RP) & $24 \mathrm{~mA}$ & $25 \mathrm{~mA}$ & $160 \mathrm{~mW}$ & $45.43 \%$ & $3.597 \mathrm{~s}$ & $0.4 \%$ & $0.575 \mathrm{~J}$ & $76.92 \%$ \\
\hline $\begin{array}{l}\text { Sleep phase (SP) } \\
\text { Power Mode } 3\end{array}$ & $0.4 \mu \mathrm{A}$ & $30 \mu \mathrm{A}$ & $0.192 \mathrm{~mW}$ & $0.0545 \%$ & $896.4 \mathrm{~s}$ & $99.59972 \%$ & $0.172 \mathrm{~J}$ & $23.01 \%$ \\
\hline
\end{tabular}

${ }^{a}$ Which corresponds to a packet of length 80 Byte and data rate of $250 \mathrm{kbit} / \mathrm{s}$.

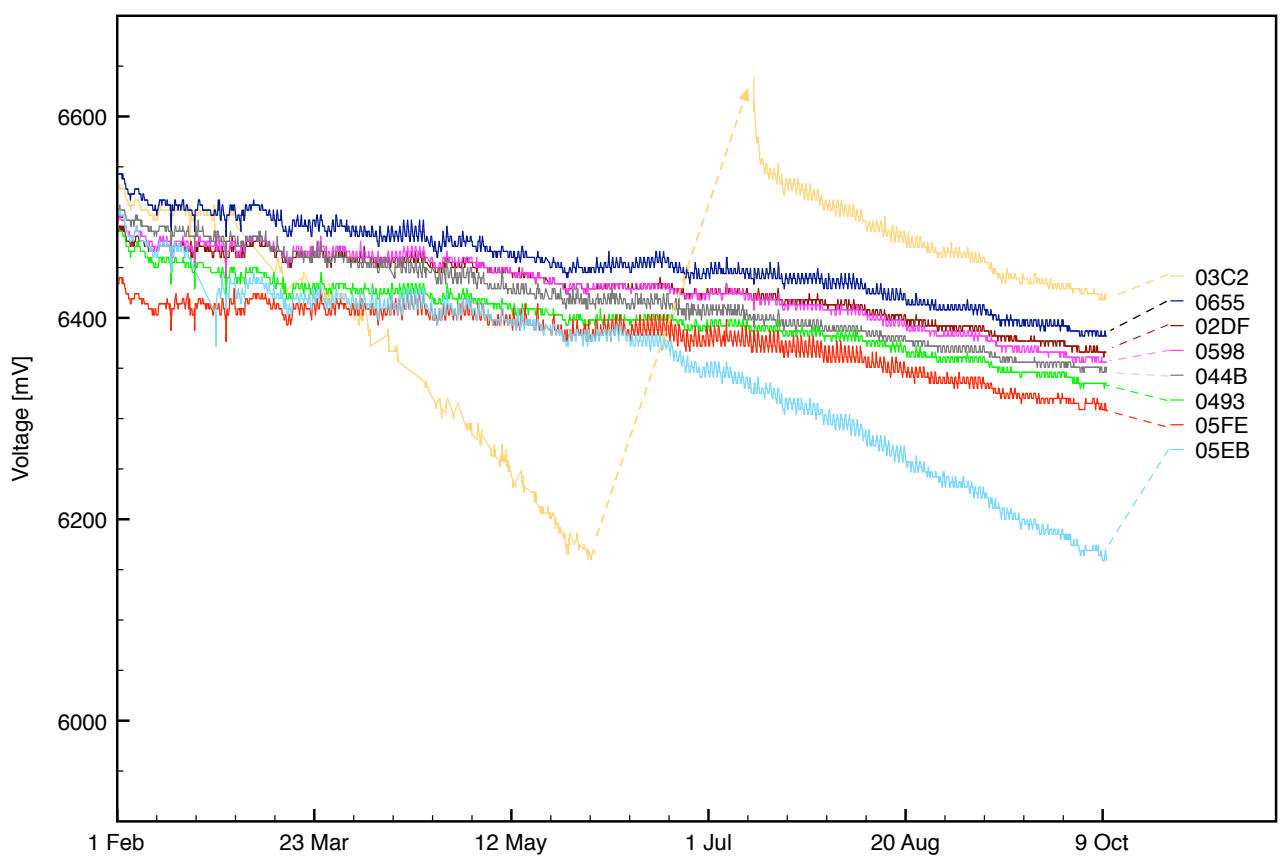

Fig. 8. Battery voltage trends during the monitoring campaign.

TABLE VI

ENERGY CONSUMPTION OF SENSORS DURING AN ACQUISITION INTERVAL $\left(T_{\mathrm{ACQ}}=15 \mathrm{MIN}\right)$

\begin{tabular}{|c|c|c|c|c|}
\hline Sensor $\downarrow$ & Current & Voltage & Energy $^{a}$ & $\frac{\text { Energy sensor }}{\text { Energy node }} \%$ \\
\hline Wire extensometer & $3 \mathrm{~mA}$ & $3 \mathrm{~V}$ & $0.0189 \mathrm{~J}$ & $2.53 \%$ \\
\hline Bar extensometer & $7 \mathrm{~mA}$ & $5 \mathrm{~V}$ & $0.0735 \mathrm{~J}$ & $9.83 \%$ \\
\hline Tiltmeter & $60 \mathrm{~mA}$ & $9 \mathrm{~V}$ & $1.134 \mathrm{~J}$ & $152 \%$ \\
\hline Soil hygrometer & $10 \mathrm{~mA}$ & $3 \mathrm{~V}$ & $0.063 \mathrm{~J}$ & $8.43 \%$ \\
\hline
\end{tabular}

${ }^{a}$ The sensor stays on for $2100 \mathrm{~ms}$, before the TP, to collect the data.

battery voltages, were collected and analyzed. The analysis of such metrics demonstrated the effectiveness of the network protocols to manage self-organization, node failures, low link quality and unexpected battery depletion, and constitutes a resource of precious information for the network designer to plan future installations.

\section{ACKNOWLEDGMENT}

Authors would like to thank Andrea Mariani for the valuable suggestions and for carefully reading the manuscript.

\section{REFERENCES}

[1] S. Kelly, N. Suryadevara, and S. Mukhopadhyay, "Towards the implementation of IoT for environmental condition monitoring in homes," IEEE Sensors Journal, vol. 13, no. 10, pp. 3846-3853, Oct 2013.

[2] M. Casoni, C. Grazia, M. Klapez, N. Patriciello, A. Amditis, and E. Sdongos, "Integration of satellite and LTE for disaster recovery," Communications Magazine, IEEE, vol. 53, no. 3, pp. 47-53, March 2015.

[3] T. Campana, M. Casoni, A. Marousis, K. Maliatsos, and A. Karagiannis, "E-SPONDER system: A new communication infrastructure for future emergency networks," in Wireless and Mobile Computing, Networking and Communications (WiMob), 2014 IEEE 10th International Conference on, Oct 2014, pp. 136-143.

[4] M. Casoni, C. Grazia, and P. Valente, "A low-latency and highthroughput scheduler for emergency and wireless networks," in Communications Workshops (ICC), 2014 IEEE International Conference on, June 2014, pp. 231-236.

[5] M. T. Lazarescu, "Design of a WSN platform for long-term environmental monitoring for IoT applications," IEEE J. on Emerging and Sel. Topics in Circ. and Sys., vol. 3, no. 1, pp. 45-54, Mar. 2013.

[6] T. Torfs, T. Sterken, S. Brebels, J. Santana, R. van den Hoven, V. Spiering, N. Bertsch, D. Trapani, and D. Zonta, "Low power wireless sensor network for building monitoring," IEEE Sensors Journal, vol. 13, no. 3, pp. 909-915, March 2013.

[7] C. See, K. V. Horoshenkov, R. Abd-Alhameed, Y.-F. Hu, and S. Tait, "A low power wireless sensor network for gully pot monitoring in urban catchments," IEEE Sensors Journal, vol. 12, no. 5, pp. 1545-1553, May 2012. 
[8] M. Meribout, "A wireless sensor network-based infrastructure for realtime and online pipeline inspection," IEEE Sensors Journal, vol. 11, no. 11, pp. 2966-2972, Nov 2011.

[9] M. Whelan, M. Gangone, and K. Janoyan, "Highway bridge assessment using an adaptive real-time wireless sensor network," IEEE Sensors Journal, vol. 9, no. 11, pp. 1405-1413, Nov 2009.

[10] M. Rodriguez-Sanchez, S. Borromeo, and J. Hernndez-Tamames, "Wireless sensor networks for conservation and monitoring cultural assets," IEEE Sensors Journal, vol. 11, no. 6, pp. 1382-1389, June 2011.

[11] H. Yang, Y. Qin, G. Feng, and H. Ci, "Online monitoring of geological $\mathrm{CO}_{2}$ storage and leakage based on wireless sensor networks," IEEE Sensors Journal, vol. 13, no. 2, pp. 556-562, Feb 2013.

[12] H. Ramamurthy, B. S. Prabhu, R. Gadh, and A. Madni, "Wireless industrial monitoring and control using a smart sensor platform," IEEE Sensors Journal, vol. 7, no. 5, pp. 611-618, May 2007.

[13] C. Ranhotigamage and S. Mukhopadhyay, "Field trials and performance monitoring of distributed solar panels using a low-cost wireless sensors network for domestic applications," IEEE Sensors Journal, vol. 11, no. 10 , pp. $2583-2590$, Oct 2011

[14] J. Matamoros, F. Fabbri, C. Anton-Haro, and D. Dardari, "On the estimation of randomly sampled 2D spatial fields under bandwidth constraints," IEEE Trans. on Wireless Commun., vol. 10, no. 12, pp. 4184-4192, December 2011.

[15] A. Giorgetti, M. Lucchi, M. Chiani, and M. Z. Win, "Throughput per pass for data aggregation from a wireless sensor network via a UAV," IEEE Trans. Aerosp. Electron. Syst., vol. 47, no. 4, pp. 2610-2626, Oct. 2011.

[16] E. Intrieri, G. Gigli, F. Mugnai, R. Fanti, and N. Casagli, "Design and implementation of a landslide early warning system," Engineering Geology, 2012.

[17] G. Gigli and N. Casagli, "Semi-automatic extraction of rock mass structural data from high resolution lidar point clouds," Int. J. of Rock Mechanics and Mining Sciences, vol. 48, no. 2, pp. 187-198, 2011

[18] A. Rosi, P. Vannocci, V. Tofani, G. Gigli, and N. Casagli, "Landslide characterization using satellite interferometry (psi), geotechnical investigations and numerical modelling: The case study of ricasoli village (italy).” Int. J. of Geosciences, vol. 4, no. 5, 2013.

[19] G. Gigli, S. Morelli, S. Fornera, and N. Casagli, "Terrestrial laser scanner and geomechanical surveys for the rapid evaluation of rock fall susceptibility scenarios," Landslides, pp. 1-14, 2012.

[20] C. Atzeni, M. Barla, M. Pieraccini, and F. Antolini, "Early warning monitoring of natural and engineered slopes with ground-based syntheticaperture radar," Rock Mechanics and Rock Engineering, vol. 48, no. 1, pp. 235-246, 2015.

[21] M. Barla and F. Antolini, "An integrated methodology for landslides' early warning systems," Landslides, vol. 13, no. 2, pp. 215-228, 2016 [Online]. Available: http://dx.doi.org/10.1007/s10346-015-0563-8

[22] G. Barla, F. Antolini, M. Barla, E. Mensi, and G. Piovano, "Monitoring of the Beauregard landslide (Aosta Valley, Italy) using advanced and conventional techniques," Engineering Geology, vol. 116, no. 3-4, pp. $218-235,2010$.

[23] R. Verdone, D. Dardari, G. Mazzini, and A. Conti, Wireless Sensor and Actuator Networks. Elsevier Science, 2008.

[24] H.-C. Lee, A. Banerjee, Y.-M. Fang, B.-J. Lee, and C.-T. King, "Design of a multifunctional wireless sensor for in-situ monitoring of debris flows," IEEE Trans. on Instr. and Meas., vol. 59, no. 11, pp. 29582967, 2010.

[25] A. Carullo, S. Corbellini, M. Parvis, and A. Vallan, "A wireless sensor network for cold-chain monitoring," IEEE Trans. on Instr. and Meas., vol. 58, no. 5, pp. 1405-1411, 2009.

[26] Y. Kim, R. G. Evans, and W. M. Iversen, "Remote sensing and control of an irrigation system using a distributed wireless sensor network," IEEE Trans. on Instr. and Meas., vol. 57, no. 7, pp. 1379-1387, 2008.

[27] M. M. Alam, O. Berder, D. Menard, T. Anger, and O. Sentieys, "A hybrid model for accurate energy analysis of WSN nodes," EURASIP J. Embedded Syst., vol. 2011, pp. 4:1-4:16, Jan. 2011. [Online]. Available: http://dx.doi.org/10.1155/2011/307079

[28] P. Mehta, D. Chander, M. Shahim, K. Tejaswi, S. Merchant, and U. Desai, "Distributed detection for landslide prediction using wireless sensor network," in Global Inform. Infrastructure Symp. IEEE, 2007, pp. $195-198$.

[29] K. Akkaya and M. Younis, "A survey on routing protocols for wireless sensor networks," Ad hoc networks, vol. 3, no. 3, pp. 325-349, 2005.

[30] C. Buratti, A. Giorgetti, and R. Verdone, "Cross layer design of an energy efficient cluster formation algorithm with carrier sensing multiple access for wireless sensor networks," EURASIP Journal on Wireless Communications and Networking, vol. 5, no. 5, pp. 672-685, Dec. 2005.
[31] M. Alam, O. Berder, D. Menard, and O. Sentieys, "TAD-MAC: Trafficaware dynamic MAC protocol for wireless body area sensor networks," IEEE J. on Emerging and Selected Topics in Circuits and Systems, vol. 2, no. 1, pp. 109-119, March 2012.

[32] J. K. Hart and K. Martinez, "Environmental sensor networks: A revolution in the earth system science?" Earth-Science Reviews, vol. 78, no. 3, pp. 177-191, 2006

[33] J.-H. Huang, Y.-Y. Chen, Y.-T. Huang, P.-Y. Lin, Y.-C. Chen, Y.-F. Lin, S.-C. Yen, P. Huang, and L.-J. Chen, "Rapid prototyping for wildlife and ecological monitoring," IEEE Systems Journal, vol. 4, no. 2, pp. 198-209, June 2010.

[34] B.-D. Lee and K.-H. Lim, "An energy-efficient hybrid data-gathering protocol based on the dynamic switching of reporting schemes in wireless sensor networks," IEEE Systems Journal, vol. 6, no. 3, pp. 378-387, Sep 2012.

[35] I. Martin, T. O’Farrell, R. Aspey, S. Edwards, T. James, P. Loskot, T. Murray, I. Rutt, N. Selmes, and T. Bauge, "A high-resolution sensor network for monitoring glacier dynamics," IEEE Sensors Journal, vol. 14, no. 11, pp. 3926-3931, Nov 2014

[36] H. Z. Kotta, K. Rantelobo, S. Tena, and G. Klau, "Wireless sensor network for landslide monitoring in nusa tenggara timur," TELKOMNIKA Ind. J. of Elec. Eng., vol. 9, no. 1, pp. 9-18, 2011.

[37] T. Fernandez-Steeger, C. Arnhardt, K. Walter, S. Haß, F. Niemeyer, B. Nakaten, S. Homfeld, K. Asch, R. Azzam, R. Bill et al., "Slewsa prototype system for flexible real time monitoring of landslides using an open spatial data infrastructure and wireless sensor networks," Geotechnologien sci. rep., vol. 13, pp. 3-15, 2009.

[38] M. V. Ramesh, S. Kumar, and P. V. Rangan, "Wireless sensor network for landslide detection." in ICWN, 2009, pp. 89-95.

[39] A. Rosi, M. Berti, N. Bicocchi, G. Castelli, A. Corsini, M. Mamei, and F. Zambonelli, "Landslide monitoring with sensor networks: experiences and lessons learnt from a real-world deployment," Int. J. of Sensor Net., vol. 10, no. 3, pp. 111-122, 2011.

[40] A. Terzis, A. Anandarajah, K. Moore, I. Wang et al., "Slip surface localization in wireless sensor networks for landslide prediction," in Int. Conf. on Info. Proc. in Sens. Net. ACM, 2006, pp. 109-116.

[41] E. A. Garich, "Wireless, automated monitoring for potential landslide hazards," Master's thesis, Texas A\& M University, 2007.

[42] A. Sheth, K. Tejaswi, P. Mehta, C. Parekh, R. Bansal, S. Merchant, T. Singh, U. B. Desai, C. A. Thekkath, and K. Toyama, "Senslide: a sensor network based landslide prediction system," in Int. Conf. on Emb. Net. Sens. Sys. ACM, 2005, pp. 280-281.

[43] R. Ohbayashi, Y. Nakajima, H. Nishikado, and S. Takayama, "Monitoring system for landslide disaster by wireless sensing node network," in First Int. Ann. Conf. SICE. IEEE, 2008, pp. 1704-1710.

[44] C. D. Hill and K. D. Sippel, "Modern Deformation Monitoring: A Multi Sensor Approach," in FIG XXII Int. Conf., Washington DC, USA, Apr. 2002, pp. 1-12.

[45] H. Kung, J. Hua, and C. Chen, "Drought forecast model and framework using wireless sensor networks," J. of Inf. Sci. and Eng., vol. 22, pp. 751-769, 2006

[46] F. Antolini, M. Barla, G. Gigli, A. Giorgetti, E. Intrieri, and N. Casagli, "Combined finite-discrete numerical modelling of runout of the Torgiovannetto di Assisi rockslide (central Italy)," ASCE Int. J. of Geomechanics, 2016. [Online]. Available: http://dx.doi.org/10.1061/ (ASCE)GM.1943-5622.0000646

[47] G. Gigli, E. Intrieri, L. Lombardi, M. Nocentini, W. Frodella, M. Balducci, L. Venanti, and N. Casagli, "Event scenario analysis for the design of rockslide countermeasures," Journal of Mountain Science, vol. 11, no. 6, pp. 1521-1530, 2014. [Online]. Available: http://dx.doi.org/10.1007/s11629-014-3164-4

[48] Winet, www.winetsrl.com, [Online].

[49] Texas Instruments, "Datasheet: A true system-on-chip solution for 2.4ghz ieee802.15.4 and zigbee applications," 2011. [Online]. Available: http://www.ti.com/lit/ds/swrs081b/swrs081b.pdf

[50] IEEE 802.15.4 Standard 2003. Part 15.4: Wireless Medium Access Control (MAC) and Physical Layer (PHY) Specifications for Low-Rate Wireless Personal Area Networks (LR-WPAN), 2003.

[51] D. Djenouri and M. Bagaa, "Synchronization protocols and implementation issues in wireless sensor networks: A review," IEEE Systems Journal, no. 99, pp. 1-11, 2014.

[52] M. Maróti, B. Kusy, G. Simon, and A. Lédeczi, "The flooding time synchronization protocol," in Proceedings of the 2Nd International Conference on Embedded Networked Sensor Systems, ser. SenSys '04. New York, NY, USA: ACM, 2004, pp. 39-49. [Online]. Available: http://doi.acm.org/10.1145/1031495.1031501 
[53] C. Otero, R. Haber, A. Peter, A. AlSayyari, and I. Kostanic, "A wireless sensor networks' analytics system for predicting performance in ondemand deployments," IEEE Systems Journal, no. 99, pp. 1-10, 2014. 